\title{
Original research \\ Immunogenicity, antigenicity and epitope mapping of Salmonella InvH protein: An in silico study
}

\author{
Behzad Dehghani ${ }^{1,,}$, Tayebeh Hashempour ${ }^{1}$, Zahra Hasanshahia ${ }^{\text {, Iraj Rasooli²,3 }}$ \\ ${ }^{1}$ Shiraz HIV/AIDS Research Center, Institute of Health, Shiraz University of Medical Sciences, Shiraz, Iran \\ 2 Department of Biology, Shahed University, Tehran, Iran \\ 3Molecular Microbiology Research Center, Shahed University, Tehran, Iran
}

\begin{abstract}
InvH is an indispensable part of T3SS-I and has a significant role in SPI-I mediated effector protein translocation. The InvH mutations have significant effects including reduced secretory and inflammatory responses that result from preventing the normal secretion of several proteins. Our team previous studies showed the capable ability of InvH to induce the humoral immune system to prevent almost all Salmonella strains infections. The current study aimed to determine all aspects of this protein using several bioinformatics tools and find the differences among all Salmonella strains. This data could pave the way for further studies about InvH protein and the production of an effective vaccine against Salmonella infections. InvH sequences for all Salmonella strains were obtained from GenBank and analyzed to determine physicochemical properties, B-Cell and T-Cell epitopes, and reliable structures. Results showed some minimal differences among Salmonella strains. B-Cell and T-Cell epitopes predicted by numerous software approved the ability of this protein to induce both humoral and cellular immune systems remarkably. This study provided a comprehensive data to determine all features of InvH protein and our results showed the ability of this protein to design a capable vaccine and the effect of amino acid changes on structure and physicochemical properties, and epitopes.
\end{abstract}

Keywords: InvH, Salmonella, Bioinformatics, SPI-I, Vaccine

\section{Introduction}

Each year 1.3 million cases of salmonellosis occur globally. Salmonella enterica (S. enterica) includes many foodborne pathogens causing salmonellosis in developing or developed countries. Patterns of salmonellosis in humans include enteric fever, gastroenteritis, bacteremia, and chronic carries. Typhoid fever is caused by Salmonella enterica serovar Typhi (S. Typhi), and paratyphoid fever is caused by Salmonella paratyphi ( $S$. paratyphi) $A, B$, and $C$. Infection is spread by contaminated food or water. Salmonella Typhimurium (S. Typhimurium) and Salmonella enterica serovar Enteritidis (S. Enteritidis) cause gastroenteritis via contaminated food or water by animal waste. Bacteremia is a serious infection associated with invasive sero-

\section{"Corresponding author:}

Behzad Dehghani, MSc

2nd floor, Voluntary Counseling and Testing Center,

Lavan Ave, Delavaran-e Basij Blvd, Khatoun Sq, Shiraz,

Fars, Iran

Tel/Fax: +98 7137386272

Email: deghanibehzad@gmail.com

https://orcid.org/0000-0002-4895-9419

Received: May, 31, 2020

Accepted: June, 10, 2020
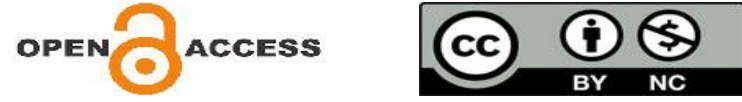
types, Salmonella cholearaesuis (S. cholearaesuis), and Salmonella Dublin (S. Dublin). Chronic carriers are major factors to spread Salmonellosis in the word [1]. Many Gram-negative bacteria have a type III secretion system. The type-III secretion system-I (T3SS-I) of S. enterica is necessary for invasion and translocation effectors proteins from bacteria into host cells. Two Salmonella pathogenicity islands of SPI1 and SPI2 encode structural components, effectors, and regulators of $\mathrm{T}_{3} \mathrm{SS} 1[2,3]$. InvH, a main part of T3SS-I, is necessary for the effective performance of Salmonella invasion of epithelial cells $[4,5]$. Deletion of invH gene is related to reduce the invasion efficiency of the bacterium compared to the wild-type [5]. Genetic analysis has shown the presentation of InvH in many Salmonella strains [6]. Immunization with recombinant InvH against $S$. Enteritidis and $S$. Typhi in animal models has shown its potential as a vaccine candidate $[7,8]$. Many bioinformatics tools have been employed to analyze the properties and features of different bacterial and viral genes [913].

This study aimed to compare experimental and bioinformatics data to find epitopes, modification sites, and structure analysis that can provide much useful information on immunogenicity and antigenicity of InvH protein.

\section{Material and Methods}

2.1. invH sequence analysis

For bioinformatics analysis, 6 sequences (full length: 444 bp, 148 amino acids) were obtained from GenBank (http://www.ncbi.nlm.nih.gov). The sequences included were accession number: CPoo9102.1 (S. Typhimurium), accession number: AOYFo1000046.1 (S. Paratyphi B), accession number: AL513382.1 (S. Typhi), accession number: NC_oo6511.1 (S. Paratyphi A), accession number: CPooo857.1 (S. Paratyphi C), and accession number: AM933172.1 (S. Enteritidis).

2.2. Amino acid changing and Phylogenetic trees

All sequences were translated, edited, and aligned using CLC sequence viewer version Beta (QIAGEN). Phylogenic trees were determined by neighbor-joining (NJ) methods 1000 times to confirm the reliability of phylogenetic trees. Signal peptide and signal peptide cleavage sites for
Gram-negative bacteria were determined using "SignalP 4.1" at (http://www.cbs.dtu.dk/services/SignalP/) [14], "Predisi" (http://www.predisi.de/predisi/startprediction) [15], and "SignalBLAST" (http://sigpep.services.came.sbg.ac.at/signalblast.html) [16].

2.3. InvH physicochemical properties

"Expasy's ProtParam" (http://expasy.org/ tools/protparam.html) was used to determine theoretical isoelectric point (pI), instability index, aliphatic index, and grand average hydropathy (GRAVY) [17-19].

2.4. Immuno-informatic prediction

"Immuneepitope" (http://tools.immuneepitope.org/tools/bcell/iedb_input) was employed to determine B-Cell epitopes positions. Chou and Fasman method was used to predict Beta-Turns, Karplus and Schulz for predicting the flexibility; Emini method was used for surface accessibility prediction, and Parker method for hydrophilicity evaluation and Bepipred method to define B-cell epitopes using crystal structures. B-cell epitopes prediction was based on hydrophilic, flexibility, surface accessibility, B-turns, antigenic, exposed surface, and Polarity scale methods using BcePred software at (http://www.imtech.res.in/raghava/bcepred) [20], and ABCpred software (http://www.imtech.res.in/raghava/abcpred/) [21]. T-cell epitope prediction was done by "SYFPEITHI" (http://www.syfpeithi.de/bin/MHCServer.dll/EpitopePrediction.htm) [22], and "immuneepitope" (http://tools.immuneepitope.org/main/tcell/) [23]. Antigenicity probability was estimated (http://www.ddg-pharmfac.net/vaxijen/VaxiJen/VaxiJen.html) using VaxiJen software [24]. Prediction of IgE epitopes was carried out by AlgPred at (http://www.imtech.res.in/raghava/algpred/submission.html) [25].

2.5. Disulfide bond prediction

Disulfide bonds position was predicted using DiANNA [26] (http://clavius.bc.edu/ clotelab/DiANNA) and SCRATCH [27] (http://scratch.proteomics.ics.uci.edu/).

2.6. Secondary structure prediction

Secondary structure was predicted and confirmed by SOPMA (http://npsa-pbil.ibcp.fr/cgibin/npsa_automat.pl?page=npsa_sopma.html), 
Phyre

(http://www.sbg.bio.ic.ac.uk/phyre) [28].

2.7. Prediction and validation of tertiary structure

\section{I-TASSER}

(http://zhanglab.ccmb.med.umich.edu/I-

TASSER) [29-32], Phyre2server (http://www.sbg.bio.ic.ac.uk/ phyre) [28], and (PS) ${ }^{2}$ Server [33] (http://ps2v2.life.nctu.edu.tw) were employed to build $3 \mathrm{D}$ structures the stereochemistry and quality of the $3 \mathrm{D}$ structures models were evaluated by Qmean [34, 35] (http://swissmodel.expasy.org/qmean/cgi/index.cgi) and Ramachandran plots were mapped by Rampage (http://mordred.bioc.cam.ac.uk/ rapper/rampage.php).

\section{Results} trees

3.1. Sequences analysis and Phylogenetic

The alignment of all sequences is shown in Figure 1. Phylogenetic tree for 6 sequences by NJ method: two main clades were shown in the tree, upper clade is divided into two clusters; in the first cluster Paratyphi A and Typhi (with 95 bootstrap score); in the second cluster Paratyphi $\mathrm{C}$ and Typhimurium are very close (with 83 bootstrap score). A clade contains Enteritidis and Paratyphi B (Figure 2). Signal peptide prediction of each software was similar for all sequences.

\subsection{Amino acid changing}

A comparison of the sequences with S. Typhi revealed only 4 amino acid residue changes. Changes at positions 36 (K-Q) and 139 (A-S) in $S$. Paratyphi C, amino acid $67(\mathrm{Q}-\mathrm{H})$ in $S$. Typhimurium, position 146 (A-S) in S. Enteritidis.

\subsection{Protparam results}

Almost the results were similar for all sequences except for pI of $S$. Paratyphi $C$ (Table 1).

\subsection{Immuno-informatics}

Immuneepitopes by Beta-Turn methods determined three positions of 65-72, 70-76 and 126132 as B-cell epitopes regions. Surface Accessibility method showed two regions (87-92 and 109-114) and flexibility method showed three regions of 124-131, 87-95 and 106-114, antigenicity method indicated region 6-16, hydrophilicity method revealed a region (107-114), Bepipred (threshold:1) method determined three regions. The regions were similar in all sequences. Seven parameters of hydrophilicity, flexibility, surface accessibility, beta turs, surface exposure and polarity were almost similar according to BcePred results (Table 2).

ABCpred results revealed a start position of 16 meric B-cell epitopes of 97, 46, 89, and 29 were similar for all sequences. Prediction of protective antigens by "VaxiJen" program was around 0.34 and was probable non-antigen for all sequences. Prediction by mapping of IgE epitopes using "AlgPred" software revealed as non-allergen for all sequences. HLA-E and HLA B27-5 T-cell epitopes results are shown in Table 3. HLA-E epitopes in all sequences were similar and HLA-B27-5 epitope regions were similar in all sequences except for $S$. Paratyphi $C$ in which region 27 in HLA-B $^{*} 27: 05$ was absent. The 8-14 meric epitopes were similar in all sequences except for $S$. Paratyphi $C$ in which region 27 in HLA$\mathrm{B}^{*} 27$ :05 was absent.

3.5. Disulfide bond prediction

Three disulfide positions $(7,48$, and 85$)$ were predicted by "DiANNA" and 4 positions $(7,16,48$, and 85 ) by "scratch". Positions $7,16,48$, and 85 were conserved for disulfide bonds. tion

3.6. Secondary and tertiary structures predic-

Percentages of secondary structures by "SOPMA" are given in Table 4. The results were almost similar in all sequences. The secondary structures are shown in Figure 3. We could not construct a valid structure for $\mathrm{InvH}$ protein using all programs.

\section{Discussion}

Cloning and molecular characterization of InvH were performed in 1993 [6]. The role of this protein in adherence and invasion to epithelial cell culture was determined in previous studies [6]. InvH is necessary for intestinal secretory and inflammatory responses, bovine macrophages lysis, and secretion of Salmonella effecter proteins by type III secretion system (TTSS). InvH is an important part of the needle complex of TTSS and is required for efficient assembly of the organelle. InvH is present in all Salmonella strains except Salmonella Heidelberg (S. Heidelberg) and Salmonella enterica subspecies arizonae (S. arizonae). InvH is highly conserved in all strains. 


\section{Dehghani et al.}

Figure 1. The alignment of all used sequences
Figure 2. Phylogenic tree for all sequences by Neighbor-Joining method with 100 bootstrap score
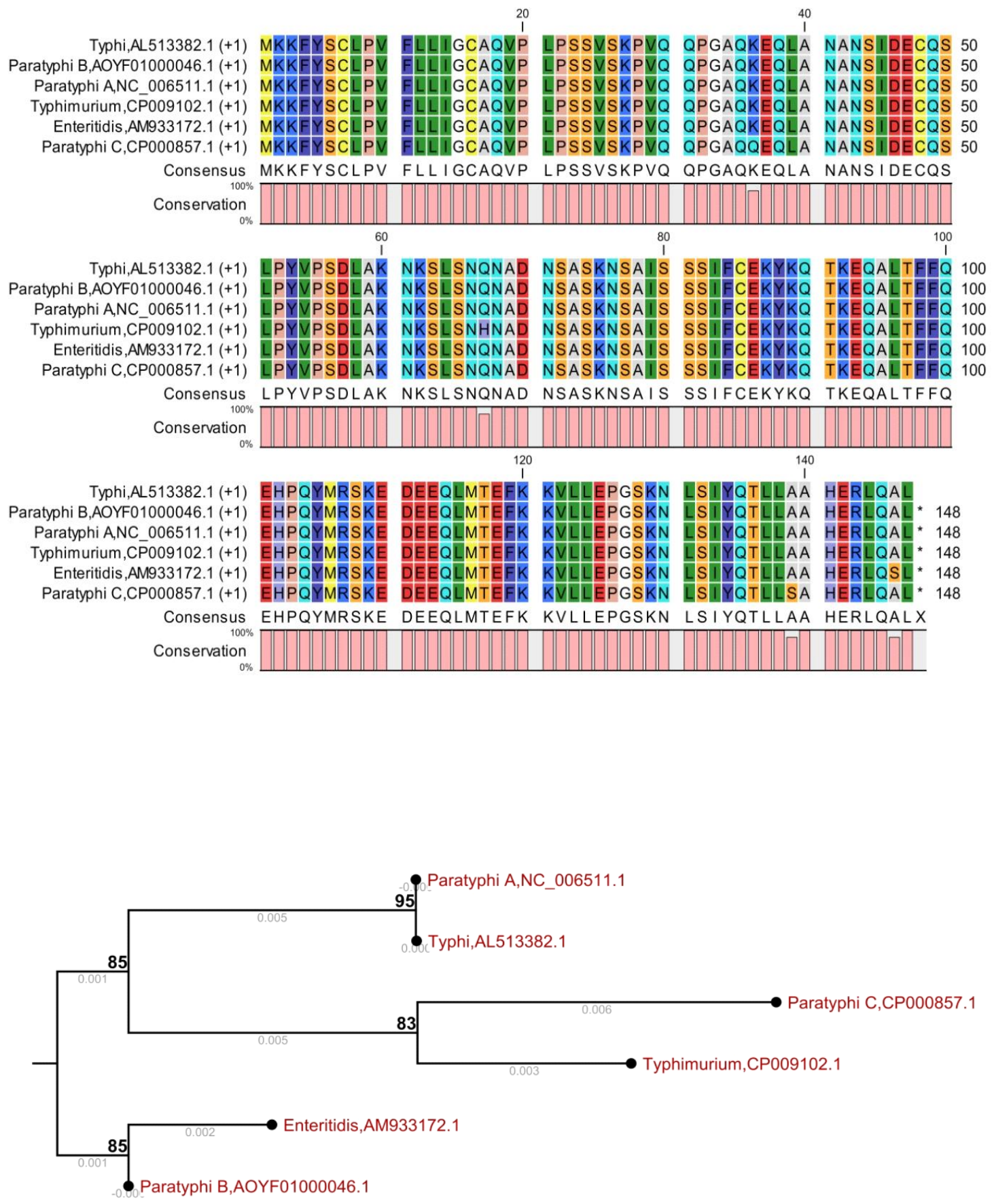


\section{Dehghani et al.}

Table 1. Physico-chemical properties of the selected sequences.

\begin{tabular}{lcccccc}
\hline Sequences & $\begin{array}{c}\text { Theoretical } \\
\text { pI }\end{array}$ & $\begin{array}{c}\text { half- } \\
\text { life } \\
\text { (hours) }\end{array}$ & $\begin{array}{c}\text { Instability in- } \\
\text { dex }\end{array}$ & $\begin{array}{c}\text { Instability clas- } \\
\text { sifies }\end{array}$ & $\begin{array}{c}\text { Aliphatic in- } \\
\text { dex }\end{array}$ & $\begin{array}{c}\text { Hydropathi- } \\
\text { city }\end{array}$ \\
\hline $\begin{array}{l}\text { Typhi- } \\
\text { murium }\end{array}$ & 7.65 & $>10$ & 59.76 & unstable & 78.37 & -0.490 \\
\hline Paratyphi B & 7.63 & $>10$ & 57.64 & unstable & 78.37 & -0.493 \\
\hline Typhi & 7.63 & $>10$ & 57.64 & unstable & 78.37 & -0.493 \\
\hline Paratyphi A & 7.63 & $>10$ & 57.64 & unstable & 78.37 & -0.493 \\
\hline Paratyphi $C$ & 6.72 & $>10$ & 60.26 & unstable & 77.69 & -0.507 \\
\hline Enteritidis & 7.63 & $>10$ & 60.63 & unstable & 77.69 & -0.510 \\
\hline
\end{tabular}

Table 2. BcePred analysis of all sequences

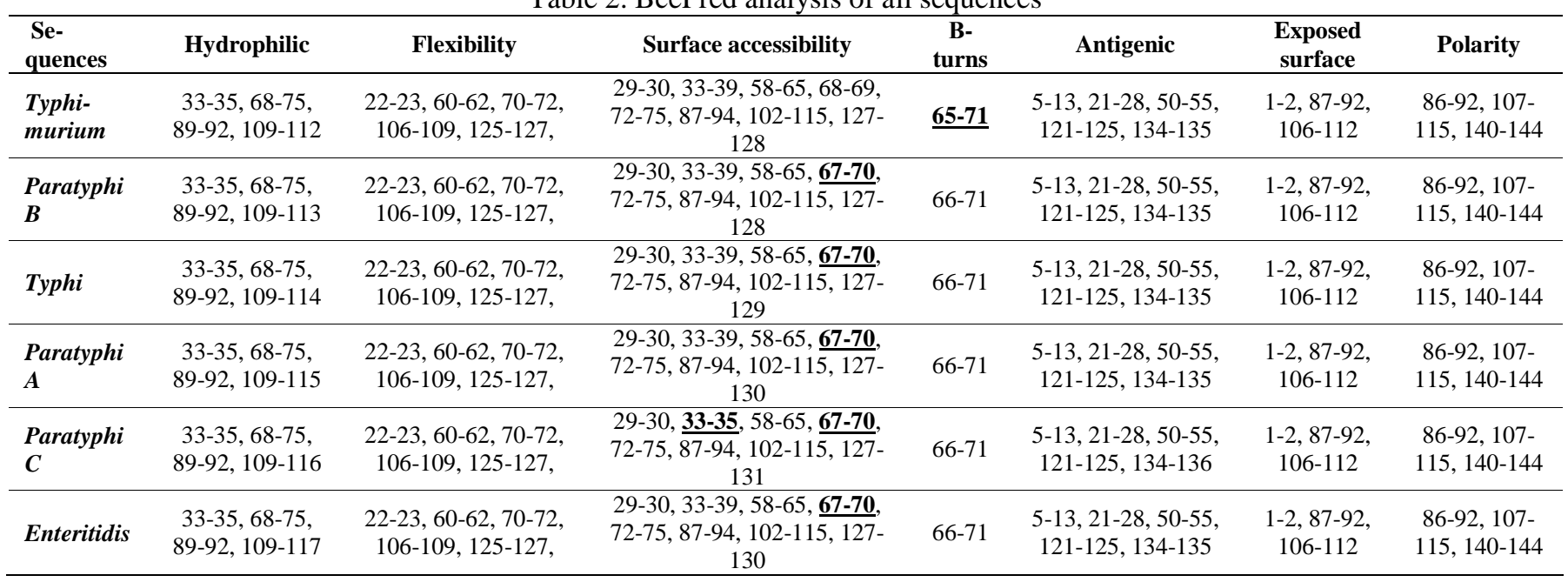

Table 3. HLA-E*01:01, HLA-B*27:05 T-cell epitope regions in selected Sequences

\begin{tabular}{lccc}
\hline HLA & Start & Length & Sequences \\
\hline HLA-E*01:01 & 35 & 8 & QKEQLANA \\
\hline HLA-E*01:01 & 66 & 8 & HPQYMRSK \\
\hline HLA-E*01:01 & 102 & 9 & PVFLLIGCA \\
\hline HLA-E*01:01 & 9 & 10 & NANSIDECQS \\
\hline HLA-E*01:01 & 41 & 10 & EKYKQTKEQA \\
\hline HLA-E*01:01 & 86 & 10 & HPQYMRSKED \\
\hline HLA-E*01:01 & 102 & 11 & HPQYMRSKEDE \\
\hline HLA-E*01:01 & 102 & 12 & QPGAQKEQLANA \\
\hline HLA-E*01:01 & 31 & 12 & HPQYMRSKEDEE \\
\hline HLA-E* $01: 01$ & 102 & 13 & NKSLSNQNADNSA \\
\hline HLA-E*01:01 & 61 & 13 & HPQYMRSKEDEEQ \\
\hline HLA-E*01:01 & 66 & 14 & DECQSLPYVPSDLA \\
\hline HLA-E*01:01 & 102 & 8 & QKEQLANA \\
\hline HLA-E*01:01 & 46 & 8 & HPQYMRSK \\
\hline HLA-E*01:01 & 55 & 9 & PVFLLIGCA \\
\hline HLA-B*27:05 & 106 & 10 & M R S K E D E E Q L \\
\hline HLA-B*27:05 & 2 & 10 & K K F Y S C L P V F \\
\hline HLA-B*27:05 & 129 & 10 & K N L S I Y Q T L L \\
\hline HLA-B*27:05 & 12 & 10 & L L I G C A Q V P L \\
\hline HLA-B*27:05 & 27 & 10 & K P V Q Q P G A Q K \\
\hline HLA-B*27:05 & 89 & 10 & K Q T K E Q A L T F \\
\hline HLA-B*27:05 & 3 & 9 & K F Y S C L P V F \\
\hline HLA-B*27:05 & 19 & 9 & V P L P S S V S K \\
\hline HLA-B*27:05 & 121 & 9 & K V L L E P G S K \\
\hline
\end{tabular}

Table 4. Percentages of the secondary structures for all sequence

\begin{tabular}{lc}
\hline Sequences & $\begin{array}{c}\text { Percentages of alpha helix, extended strand, beta turn, } \\
\text { random coil }\end{array}$ \\
\hline $\begin{array}{l}\text { Typhi- } \\
\text { murium }\end{array}$ & $50.34,4.76,2.72,42.18$ \\
\hline Paratyphi $B$ & $51.02,4.76,2.72,41.50$ \\
\hline Typhi & $51.02,4.76,2.72,41.50$ \\
\hline Paratyphi $A$ & $51.02,4.76,2.72,41.50$ \\
\hline Paratyphi $C$ & $51.02,4.76,2.72,41.50$ \\
\hline Enteritidis & $51.02,4.76,2.72,41.50$ \\
\hline
\end{tabular}


Figure 3. Secondary structures of all sequences predicted and validated by "SOMPA". Blue: helix, Red: strand, Purple: coil and Green: beta turn. A: Typhimurium, B: Paratyphi B, C: Typhi, D: Paratyphi A, E: Paratyphi C, F: Enteritidis
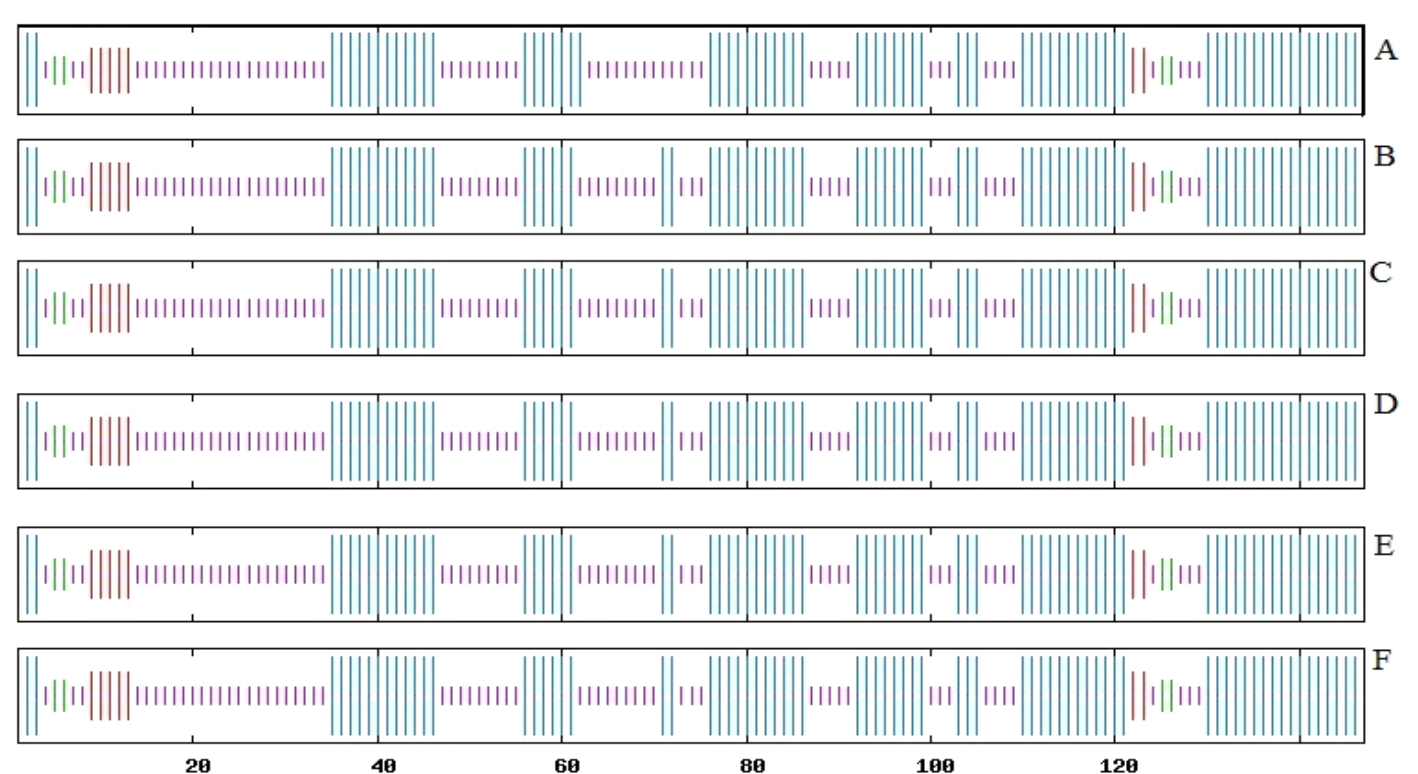

Three residual changes were determined between S. typhimurium and S. choleraesuis [6]. In this study, we found 4 residual changes and the phylogenic tree showed high similarity between 6 sequences because of the similarity in InvH sequences.

S. Typhimurium, S. Paratyphi A, S. Paratyphi B, S. Paratyphi C, S. Typhi, and S. Enteritidis are important in foodborne diseases. In our previous studies, the presentation of InvH on bacterial surface and antibody interaction with this protein was validated.

Theoretical pI for all sequences was 7.63 except for $S$. Paratyphi $C$, which was 6.72 . This could be attributed to a change in acidic amino acid to a basic one (lysine to glutamine 36 ).

Instability index provided estimated protein stability of 77.7 to 78.3 in a test tube that showed this protein is an unstable protein (Instability index of $<40$ is regarded).

The aliphatic index was 78 for all sequences which is a positive factor for the thermostability of globular proteins and depends on aliphatic side chains (alanine, valine, isoleucine, and leucine).

The aliphatic index of a protein is defined as the relative volume occupied by aliphatic side chains (alanine, valine, isoleucine, and leucine) and it may be regarded as a positive factor for the increase of the thermostability of globular proteins. The aliphatic index of a protein is calcu- lated according to the following formula: Aliphatic index $=\mathrm{X}(\mathrm{Ala})+\mathrm{a} * \mathrm{X}(\mathrm{Val})+\mathrm{b} *(\mathrm{X}(\mathrm{Ile})+$ $\mathrm{X}(\mathrm{Leu})$ ) where $\mathrm{X}(\mathrm{Ala}), \mathrm{X}(\mathrm{Val}), \mathrm{X}(\mathrm{Ile})$, and $\mathrm{X}(\mathrm{Leu})$ are mole percent (100 X mole fraction) of alanine, valine, isoleucine, and leucine. The coefficients a and $b$ are the relative volume of valine side chain $(a=2.9)$ and of Leu/Ile side chains $(b=3.9)$ to the side chain of alanine.

Hydropathicity index was around -0.6 for all sequences shows hydrophilicity of the protein. Hydrophilicity of protein parts is a positive patent for immune proteins because these parts are almost exposed and have the potential of interaction with immunoglobulins.

Signal peptide cleavage sites and signal peptide region prediction by three programs shows three (19, 26, and 15) different sites. "Predisi" results were more reliable and similar to experimental results. The mutation in nucleotide 106 (A to C) brought about amino acid change (K-Q 36) in S. Paratyphi C, nucleotide 199 (G to T) brought about amino acid change (Q-H 67) in S. Typhimurium. The mutation in nucleotide 415 ( $\mathrm{G}$ to $\mathrm{T}$ ) changed the amino acid 139 from A-S in S. Paratyphi $C$, and that in nucleotide 436 ( $\mathrm{G}$ to $\mathrm{T}$ ) changed the amino acid $67(\mathrm{Q}-\mathrm{H})$ in $S$. Enteritidis.

B-cell epitopes prediction by "Immuneepitope" and six other methods showed some potent similar regions. A combination of the results of all methods showed four potent B-cell regions (65- 
76, 87-95, 106-114, and 124-132). B-cell epitopes by BcePred showed similar regions in all sequences. ABCpred regions (29-45, 46-62, 89-105, and 97-113) were similar in all sequences. Immuneepitope, BcePred, and ABCpred showed the most potent B-cell epitope of 86-115 for InvH protein. Region 86-115 contains the best potential in terms of hydrophilicity, flexibility, surface accessibility, surface exposed position, polarity, and linear B-cell epitopes prediction. This region contains almost alpha helix and random coil structures.

Immunization of mice by recombinant InvH showed a significant rise of IgG and humoral response and protective immunization against $S$. Typhi and S. Enteritidis $[7,8]$ confirming our immune-informatics analysis. "VaxiJen" showed InvH protein as probable non-antigen. AlgPred determined InvH as non-allergen.

HLA-E binds nonamer peptides derived from bacterial proteins and triggers CD8-mediated lysis and IFN-production when exposed to infected targets, raising the possibility that this novel effector mechanism might contribute to host defense against intracellular bacterial infections. Salmonella OMP peptides binding to HLA$B * 27: 05$ were identified to modulate the host-microbe interaction and HLA-B27 confers a very strong genetic predisposition to the development of reactive arthritis after infection by bacteria such as $S$. Typhimurium [36].

Our results showed 9 HLA-B*27:05 and 15 HLA-E T-cell epitopes regions. We, however, found two 9 meric T-cell epitopes (102-111 and 55-64) that indicated a good potential of InvH to stimulate cell-mediated immune system.

Disulfide bonds are important in determining the functional linkages and stability of proteins. SS bonds were analyzed using two reliable programs. Four cysteine amino acids making two disulfide bonds in InvH contributed to protein stability. Amino acid 16, 48 were located on coil structure, 7 on strand structure, and 58 was on helix structure and any of them were not located on selected B-cell epitope region (86-115).

Secondary structure in InvH protein contained almost alpha helix and random coil. Results of all the sequences were similar except for $S$. Typhimurium in which region $60-80$ helix was changed to random coil because of the change in amino acid (Q-H 67). We, however, could not achieve a proper $3 \mathrm{D}$ model for $\mathrm{InvH}$, in spite of using three popular and reliable programs.

The limited number of sequences and the number of bioinformatics software employed are the main limitation of the present study. In addition, other Salmonella strains can be added for further studies.

To sum up, it can be concluded that the findings of the present study provided valuable data about InvH protein which can be used to design a novel vaccine against all Salmonella strains as well as all physicochemical features of this protein were defined which can be useful to express this protein in available hosts.

\section{Acknowledgements}

The authors wish to thank Farahnaz Dehghani for her kind support.

\section{Author Contributions}

Conception or design of the work: BD and IR; Data collection: $\mathrm{ZH}$, TH; Data analysis and interpretation: $\mathrm{BD}, \mathrm{ZH}$; Drafting the article: $\mathrm{BD}$, IR; Critical revision of the article: $\mathrm{BD}, \mathrm{TH}$, all authors read and approved the final version of manuscript.

\section{Conflict of Interests}

Authors declare there is no conflict of interest.

\section{Ethical declarations \\ Not applicable \\ Funding resource \\ None}

\section{References}

1. Pui C, Wong W, Chai L, Tunung R, Jeyaletchumi P, Hidayah N, et al. Salmonella: A foodborne pathogen. Int. Food Res. J. 2011; 18(2):465-473.

2. Coburn B, Sekirov I, Finlay BB. Type III secretion systems and disease. Clin Microbiol Rev. 2007; 20(4):535-49.

3. Cordero-Alba M, Bernal-Bayard J, Ramos-Morales F. SrfJ, a Salmonella type III secretion system effector regulated by PhoP, RcsB, and IolR. J Bacteriol. 2012; 194(16):4226-36.

4.Ghosh P. Process of protein transport by the type III secretion system. Microbiol Mol Biol Rev. 2004; 68(4):771-95.

5. Pati NB, Vishwakarma V, Jaiswal S, Periaswamy B, Hardt W-D, Suar M. Deletion of invH gene in Salmonella enterica serovar Typhimurium limits the secretion of Sip effector proteins. Microbes Infect. 2013; 15(1):66-73.

6. Altmeyer RM, McNern JK, Bossio J, Rosenshine I, Finlay BB, Galán JE. Cloning and molecular characterization of a gene 


\section{Dehghani et al.}

involved in Salmonella adherence and invasion of cultured epithelial cells. Mol Microbiol. 1993; 7(1):89-98.

7. Dehghani B, Rasooli I, Jalali-Nadoushan M, Owlia P, Rasooli Z. Immunoprotectivity of Salmonella enterica serovar Enteritidis virulence protein, InvH, against Salmonellatyphi. Iran J Basic Med Sci. 2014; 17(8):560.

8. Dehghani B, Rasooli I, Gargari SLM, Nadooshan MRJ, Owlia P, Nazarian S. Immunogenicity of Salmonella enterica serovar Enteritidis virulence protein, InvH, and cross-reactivity of its antisera with Salmonella strains. Microbiol Res. 2013; 168(2):8490.

9. Behzad D, Zahra H, Tayebeh H, Parvin Afsar K. Subtype Classification by Polymerase and Gag Genes of HIV-1 Iranian Sequences Registered in the NCBI GenBank. Curr Proteomics. 2020; 17:1-12.

10. Tayebeh H, Behzad D, Zahra M, Tahereh A, Zahra H, Javad M, et al. Association of Mutations in the NS5A-PKRBD Region and IFNL4 Genotypes with Hepatitis C Interferon Responsiveness and its Functional and Structural Analysis. Curr Proteomics. 2020; 17:1-12.

11. Dehghani B, Hasanshahi Z, Hashempour T. HIV Capsid and Protease, New Targets of Melittin. Int J Pept Res Ther. 2020. [In press]. doi: https://doi.org/10.1007/s10989-019-10002-9

12. Dehghani B, Hasanshahi Z, Hashempour T, Motamedifar M. The possible regions to design Human Papilloma Viruses vaccine in Iranian L1 protein. Biologia. 2020; 75(5):749-59.

13. Hashempour T, Dehghani B, Mousavi Z, Yahaghi M, Hasanshahi Z, Moayedi J, et al. Evaluating Drug Resistant Mutations to HCV NS3 Protease Inhibitors in Iranian Naïve Patients. Int $J$ Pept Res Ther. 2019. [In press]. doi: https://doi.org/10.1007/s10989-019-09957-6

14. Petersen TN, Brunak S, Von Heijne G, Nielsen H. SignalP 4.0: discriminating signal peptides from transmembrane regions. Nat Methods. 2011; 8(10):785.

15. Hiller K, Grote A, Scheer M, Münch R, Jahn D. PrediSi: prediction of signal peptides and their cleavage positions. Nucleic Acid Res. 2004; 32:W375-W9.

16. Frank K, Sippl MJ. High-performance signal peptide prediction based on sequence alignment techniques. Bioinformatics. 2008; 24(19):2172-6.

17. Gasteiger E, Hoogland C, Gattiker A, Wilkins MR, Appel RD, Bairoch A. Protein identification and analysis tools on the ExPASy server. The proteomics protocols handbook: Springer; 2005. p. 571-607.

18. Musavi Z, Hashempour T, Moayedi J, Dehghani B, Ghassabi F, Hallaji M, et al. Antibody development to $\mathrm{HCV}$ alternate reading frame protein in liver transplant candidate and its computational analysis. Curr Proteomics. 2020; 17(2):154-70.

19. Dehghani B, Hashempour T, Hasanshahi Z. Interaction of human herpesvirus 8 viral interleukin-6 with human interleukin6 receptor using in silico approach: the potential role in HHV-8 pathogenesis. Curr Proteomics. 2020; 17(2):107-16.

20. Saha S, Raghava GPS. (2004) BcePred: Prediction of Continuous B-Cell Epitopes in Antigenic Sequences Using Physico-chemical Properties. In: Nicosia G, Cutello V, Bentley PJ,
Timmis J. (eds) Artificial Immune Systems. ICARIS 2004. Lecture Notes in Computer Science, vol 3239. Springer, Berlin, Heidelberg. 21. Saha S, Raghava GPS. Prediction of continuous B-cell epitopes in an antigen using recurrent neural network. Proteins. 2006; 65(1):40-8.

22. Rammensee H-G, Bachmann J, Emmerich NPN, Bachor OA, Stevanović S. SYFPEITHI: database for MHC ligands and peptide motifs. Immunogenetics. 1999; 50(3-4):213-9.

23. Lundegaard C, Lund O, Nielsen M. Accurate approximation method for prediction of class I MHC affinities for peptides of length 8,10 and 11 using prediction tools trained on 9mers. Bioinformatics. 2008; 24(11):1397-8.

24. Doytchinova IA, Flower DR. VaxiJen: a server for prediction of protective antigens, tumour antigens and subunit vaccines. BMC Bioinformatics. 2007; 8(1):4.

25. Saha S, Raghava G. AlgPred: prediction of allergenic proteins and mapping of IgE epitopes. Nucleic Acid Res. 2006;34:W202W9.

26. Ferrè F, Clote P. DiANNA: a web server for disulfide connectivity prediction. Nucleic Acid Res. 2005; 33:W230-W2.

27. Cheng J, Randall AZ, Sweredoski MJ, Baldi P. SCRATCH: a protein structure and structural feature prediction server. Nucleic Acid Res. 2005; 33:W72-W6.

28. Kelley LA, Sternberg MJ. Protein structure prediction on the Web: a case study using the Phyre server. Nat Protoc. 2009; 4(3):363.

29. Roy A, Kucukural A, Zhang Y. I-TASSER: a unified platform for automated protein structure and function prediction. Nat Protoc. 2010; 5(4):725.

30. Dehghani B, Hashempour T, Hasanshahi Z, Moayedi J. Bioinformatics analysis of domain 1 of HCV-core protein: Iran. Int J Pept Res Ther. 2020; 26(1):303-20.

31. Dehghani B, Hashempour T, Hasanshahi Z. Using immunoinformatics and structural approaches to design a novel HHV8 vaccine. Int J Pept Res Ther. 2020; 26(1):321-31.

32. Dehghani B, Ghasabi F, HashempoorT, Joulaei H, Hasanshahi Z, Halaji M, et al. Functional and structural characterization of Ebola virus glycoprotein (1976-2015)-An in silico study. Int J Biomath. 2017; 10(08):1750108.

33. Chen CC, Hwang JK, Yang JM. (PS)2: Protein Structure Prediction Server. Nucleic Acids Res. 2006; 34:W152-7.

34. Benkert P, Tosatto SC, Schomburg D. QMEAN: A comprehensive scoring function for model quality assessment. Proteins. 2008; 71(1):261-77.

35. Moattari A, Dehghani B, Khodadad N, Tavakoli F. In silico functional and structural characterization of $\mathrm{H} 1 \mathrm{~N} 1$ influenza a viruses hemagglutinin, 2010-2013, Shiraz, Iran. Acta Biotheor. 2015; 63(2):183-202.

36. Singh AK, Aggarwal A, Chaurasia S, Misra R. Identification of immunogenic HLA-B* 27: 05 binding peptides of salmonella outer membrane protein in patients with reactive arthritis and undifferentiated spondyloarthropathy. J Rheumatol. 2013; 4O(2):173-85. 